\title{
Comparative study of fentanyl and morphine in addition to hyperbaric or isobaric bupivacaine in combined spinal anaesthesia for caesarean section
}

\author{
Ayten Saracoglu ${ }^{1}$, Kemal T. Saracoglu $\mathrm{u}^{1}$, Zeynep Eti ${ }^{2}$
}

\author{
1Division of Pain Medicine, Department of Anaesthesiology, Central Education \\ and Research Hospital, Erzurum, Turkey \\ 2Division of Pain Medicine, Department of Anaesthesiology, Medical School \\ of Marmara University, Istanbul, Turkey
}

\author{
Submitted: 6 July 2010 \\ Accepted: 12 September 2010
}

Arch Med Sci 2011; 7, 4: 694-699

DOI: 10.5114/aoms.2011.24141

Copyright $\odot 2011$ Termedia \& Banach

\begin{abstract}
Introduction: The aim of our study was to compare the effects of isobaric and hyperbaric bupivacaine combined with morphine or fentanyl in patients undergoing caesarean section. We assessed quality and spread of analgesia and anaesthesia, postoperative analgesic requirement and side effects.

Material and methods: Hundred patients with American Society of Anesthesiologists physical status (ASA) I-II, age 18 to 40 years, were randomized to 4 groups. The intrathecal solutions were isobaric bupivacaine + morphine (group A), isobaric bupivacaine + fentanyl (group B), heavy bupivacaine + + morphine (group C) and heavy bupivacaine + fentanyl (group D). Mean arterial pressure, heart rate, oxygen saturation, ephedrine consumption, analgesic requirement time and additional analgesic needs were recorded.

Results: The $1^{\text {st }}$ min value of mean arterial pressure was the lowest one in all groups. Heart rate decreased significantly in group $A$ at the $10^{\text {th }}$ min but not in the other groups. The decrease of visual analogue scale (VAS) pain scores began in the groups after the $4^{\text {th }}$ postoperative $h(p<0.05)$ and the VAS value of group $B$ at the $8^{\text {th }} \mathrm{h}$ was significantly higher than the other groups. The first analgesic requirement time in the postoperative period was longer in patients who had intrathecal morphine than those who had fentanyl. The duration of analgesia with isobaric bupivacaine and morphine was the longest one.

Conclusions: We concluded that intrathecal morphine provides a long duration of postoperative analgesia but the duration gets longer when it is combined with plain bupivacaine instead of heavy bupivacaine.
\end{abstract}

Key words: opioid, combined spinal, caesarean, analgesia, bupivacaine.

\section{Introduction}

Combined spinal epidural anaesthesia (CSEA) is nowadays a popular technique in obstetric and gynaecological surgery, including caesarean delivery [1]. Combined spinal epidural anaesthesia involves intentional subarachnoid blockade and epidural catheter placement during the same procedure. Advantages include rapid onset, profound neuraxial block, the ability to titrate or prolong blockade, lower total drug dosages, higher level of patient satisfaction and decreased incidence of accidental dural puncture $[2,3]$. Sensorial blockade level is important for successful anaesthesia. The

\author{
Corresponding author: \\ Kemal T. Saracoglu MD \\ Bolge Egitim ve Arastirma \\ Hastanesi Ameliyathanesi \\ Cat Yolu Yildizkent Palandoken \\ 25100 Erzurum, Turkey \\ Phone: +90 5385478620 \\ E-mail: \\ aytentolgasaracoglu@mynet.com
}


factors that effect the sensorial blockade are baricity, dose, volume and concentration of local anaesthetics, barbotage and demographic properties of patients such as weight, height or age. Baricity is the most important factor for local anaesthetic distribution. According to baricity conception, isobaric solutions remain in the proximity of the injection site and hyperbaric solutions gravitate to dependent areas [4]. The duration of spinal anaesthesia is related to the dose of hyperbaric or isobaric local anaesthetics administered. The relationship between dose, volume, and concentration of intrathecal local anaesthetics and the sensory spread of anaesthesia has been extensively studied using isobaric local anaesthetics [5].

Bupivacaine is an amide local anaesthetic with a moderately rapid onset and long duration of action. Isobaric bupivacaine is formulated using normal saline as a diluent with a specific gravity of 1000 at $37^{\circ} \mathrm{C}$. Hyperbaric bupivacaine in $8 \%$ glucose has a specific gravity of 1021 at $37^{\circ} \mathrm{C}$. Both isobaric and hyperbaric bupivacaine have been used for spinal anaesthesia with good results [6]. Hyperbaric bupivacaine attains higher sensory levels of intrathecal anaesthesia than equal doses of plain bupivacaine. Plain bupivacaine is unpredictable in its behaviour, often spreading to cervical dermatomal levels [7]. The addition of opioids to local anaesthetics for spinal anaesthesia is increasingly common both to enhance anaesthesia and to provide postoperative analgesia [8]. The dose of local anaesthetics can be reduced by adding opioids. On the other hand, the incidence of side effects decreases because of lower doses. Small doses of opioids administered to the central nervous system provide adequate analgesia, reducing the side effects of intravenous analgesic administration. Pruritus, nausea and vomiting or respiratory depression are some examples [9]. This adjuvant analgesic technique is expected to decrease postoperative pain intensity and opioid requirements and to speed up recovery. Intrathecal morphine, which is less hydrophobic than other opioids, has a longer residence time in the cerebrospinal fluid and provides excellent postoperative analgesia [10]. Decades of clinical use demonstrate clear differences between morphine and mu-opioid receptor agonist fentanyl in time course and dermatomal extent of analgesia [11]. According to experimental studies [12], intrathecally administered fentanyl is cleared from cerebrospinal fluid within a relatively short distance, whereas morphine extends to a much greater dermatomal distribution.

The aim of our study was to compare the effects of the baricity of bupivacaine combined with morphine or fentanyl for spinal anaesthesia in patients undergoing caesarean section. We assessed the quality and spread of analgesia and anaesthesia, postoperative first analgesic requirement time and side effects.

\section{Material and methods}

After approval by our local research and the regional ethics committee and informed patient written consent, 100 unpremedicated patients with ASA physical status I-II, aged 18 to 40 years, were included in this randomized, double-blind, prospective study. The patients were scheduled for elective caesarean section under spinal anaesthesia and they also had epidural analgesia after the operation for postoperative analgesia with a CSEA technique. Extremely obese parturients (body mass index $-\mathrm{BMI}>40 \mathrm{~kg} / \mathrm{m}^{2}$ ), and patients who refused to join the study, had contraindications for neuraxial block, had cardiovascular or respiratory disorders or had an unsuccessful spinal block were excluded from the study. The anaesthetists who performed the CSEA collected the data and the patients were blinded to the solutions.

Before the spinal block, a peripheral venous cannula was placed and i.v. infusion of $6 \%$ hydroxyethyl starch 130/0.4 in $0.9 \%$ sodium chloride solution $10 \mathrm{ml} / \mathrm{kg}$ was started for fluid loading. No vasopressors were administered before the procedure.

In all patients, using an aseptic technique in the sitting position, $2 \mathrm{ml}$ of $1 \%$ lidocaine was infiltrated into the subcutaneous area of the lumbar 4-5 interspace. The epidural space was identified by the loss of resistance technique with $0.9 \%$ saline solution using an 18-gauge Tuohy needle. A spinal tap was performed using a 27-gauge Quincke spinal needle which was introduced inside the Tuohy needle. After injection of study drugs, a 20G soft tip epidural catheter with 3 lateral eyes (Perifix ${ }^{\circledR}$, B Braun Melsungen Ag, D-Melsungen) was introduced $6 \mathrm{~cm}$ into the epidural space for postoperative analgesia in all patients.

Patients were randomized to 4 groups according to the intrathecal drugs injected by using computergenerated randomization table as:

- group A: intrathecal 0.5\% isobaric bupivacaine $9 \mathrm{mg}+$ morphine sulfate $200 \mu \mathrm{g}$,

- group B: intrathecal 0.5\% isobaric bupivacaine $9 \mathrm{mg}+$ fentanyl $25 \mu \mathrm{g}$,

- group C: intrathecal $0.5 \%$ heavy bupivacaine $12.5 \mathrm{mg}+$ morphine sulfate $200 \mu \mathrm{g}$,

- group D: intrathecal $0.5 \%$ heavy bupivacaine $12.5 \mathrm{mg}+$ fentanyl $25 \mu \mathrm{g}$.

Sensory blockade was assessed using the cold sensation test on each side of the midclavicular line. The degree of motor blockade was scored from 0 to 3 by the Bromage scale ( 0 - no motor effects, $1-$ a decrease in muscle strength with ability to 
move the leg against pressure, 2 - inability to move the leg against pressure without complete paralysis, and 3 - unable to move feet or knees) at $1 \mathrm{~h}, 2 \mathrm{~h}$, $4 \mathrm{~h}, 8 \mathrm{~h}, 12 \mathrm{~h}$ and $24 \mathrm{~h}$.

Non-invasive mean arterial blood pressure was recorded with 1 min intervals. Heart rate, arterial oxygen saturation by pulse oximetry and respiratory rate were continuously measured. Finally, total ephedrine consumption, the duration of operation, first analgesic requirement time and additional analgesic needs were recorded. Pain scores were assessed by VAS. A score of 0 meant 'no pain' and 100 meant 'the worst possible pain'. The patients who had a postoperative VAS score above 30 were administered a bolus dose of epidural solution in a volume of 9 cc via an epidural catheter. The epidural bolus solution contained 3.6 cc of $0.5 \%$ isobaric bupivacaine, $3 \mathrm{mg}$ of morphine sulfate and $2.4 \mathrm{cc}$ of saline. Thereby the concentration of bupivacaine was $0.2 \%$. All patients received at least one time analgesic in the first postoperative $24 \mathrm{~h}$. Then the number of patients who required the epidural analgesic a second time was recorded as additional analgesic need. The additional analgesic need was provided by epidural bolus solution. The epidural catheter was removed at the end of the $24^{\text {th }} \mathrm{h}$. In all patients $75 \mathrm{mg}$ of diclophenac sodium intramuscularly was administered every $12 \mathrm{~h}$.

Maternal hypotension was defined as systolic arterial pressure $<90 \mathrm{mmHg}$ or when decreased more than $25 \%$ from the baseline value and was treated by increasing the intravenous infusion rate and, if necessary, administering ephedrine $10 \mathrm{mg}$ i.v. Patients who required fluids and vasopressors were considered to have hypotension. $0.01 \mathrm{mg} / \mathrm{kg}$ of $i . v$. atropine was administered for a heart rate below 45 beats/min.

Sedation and nausea and vomiting were also recorded postoperatively. The level of sedation was assessed with the Ramsey scale (1 - anxious, agitated, restless, 2 - cooperative, oriented, tranquil, 3 - responds to comments only, 4 - brisk response to light glabellar tap or loud noise, 5 - sluggish response to light glabellar tap or loud noise, 6 - no response). Postoperative nausea and vomiting was assessed with a two-point scale (0 - no episode, 1 - at least one episode).

\section{Statistical analysis}

Statistical Package for Social Sciences (SPSS) for Windows 10.0 programme was used for statistical analysis. The main outcome of our study was a $25 \%$ increase in the first analgesic requirement time. Group size was selected by using proportions sample size estimates ( $\alpha$ : 0.05, $\beta$ : 0.09). The values were expressed as mean \pm SD.

One-way ANOVA parametric test was used for comparisons between groups for normal distributed parameters; the post-hoc test was Tukey Kramer. Kruskal Wallis and Mann-Whitney $U$ statistical tests were used for non-normally distributed parameters; the post-hoc test was Dunn. Qualitative data were compared using the $\chi^{2}$ test $(p<0.05)$. A $p>0.05$ was considered significant.

\section{Results}

There were no differences between groups regarding their age, weight, height or duration of surgery $(p>0.05)$ (Table I). One patient in group B was excluded because of an accidental dural puncture by the Tuohy needle and 1 patient in group D was excluded because of unsuccessful spinal blockade. Another 2 patients were included and a total of 100 patients were analysed.

The mean arterial pressure decreased significantly at the $1^{\text {st }} \mathrm{min}$ in all groups but no statistically significant difference was seen between groups, as shown in Figure $1(p>0.05)$. Heart rate decreased significantly in group A at the $10^{\text {th }}$ min but not in the other groups $(p<0.05)$ (Figure 2). There was no statistically significant difference between groups according to the incidence of nausea and vomiting or ephedrine requirement (Tables II and III).

The main spread of analgesia and anaesthesia was similar between groups $(p>0.05)$. Patients' assessment of sensory levels after spinal anaesthesia changed within the range of T4 to T12. Bromage scales at the initial period were 3 for all patients; the quality of spinal analgesia was sufficient and no patient required analgesic supplementation during the surgery. None of the patients required additional medication throughout the operation or during data collection. There were

Table I. Patient demographics and duration of surgery (mean \pm SD)

\begin{tabular}{|lllll|}
\hline Parameters & Group A & Group B & Group C & Group D \\
\hline Age [year] & $26 \pm 6.06$ & $25.92 \pm 4.22$ & $25.92 \pm 4.68$ & $27.92 \pm 4.58$ \\
\hline Weight [kg] & $73.76 \pm 7.16$ & $72.52 \pm 7.68$ & $72.84 \pm 8.57$ & $75 \pm 8.15$ \\
\hline Duration of surgery [min] & $31.56 \pm 6.69$ & $29.52 \pm 6.38$ & $26.96 \pm 5.41$ & $30 \pm 6.45$ \\
\hline Height [cm] & $163.44 \pm 4.01$ & $162.88 \pm 5.16$ & $163.12 \pm 4.12$ & $163.64 \pm 3.04$ \\
\hline
\end{tabular}

$p>0.05$, group $A(n=25)$ : intrathecal $0.5 \%$ isobaric bupivacaine $9 \mathrm{mg}+$ morphine sulfate $200 \mu \mathrm{g}$, group $B(n=25)$ : intrathecal $0.5 \%$ isobaric bupivacaine $9 \mathrm{mg}+$ fentanyl $25 \mu \mathrm{g}$, group C $(n=25)$ : intrathecal $0.5 \%$ heavy bupivacaine $12.5 \mathrm{mg}+$ morphine sulfate $200 \mu \mathrm{g}$, group $D(n=25)$ : intrathecal $0.5 \%$ heavy bupivacaine $12.5 \mathrm{mg}+$ fentanyl $25 \mu \mathrm{g}$ 


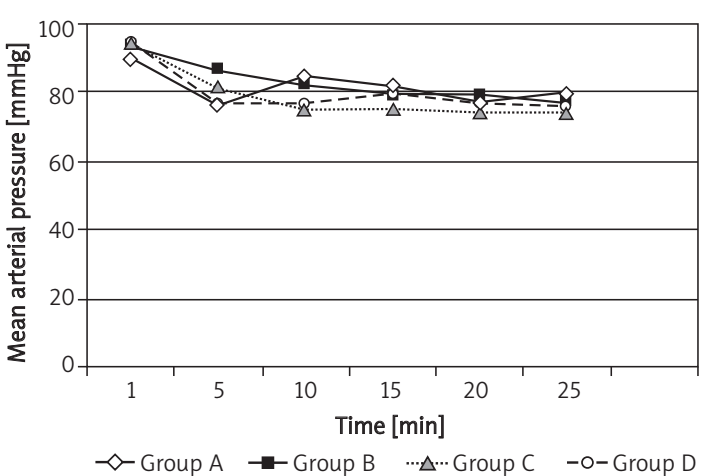

Figure 1. Mean arterial pressure values after spinal blockade

no side effects or complications observed due to the procedure. No postdural puncture headache or neurological deficits were observed.

The decrease of VAS scores began in the groups after the postoperative $4^{\text {th }} \mathrm{h}(p<0.05)$ and VAS values of group $B$ at the $8^{\text {th }} \mathrm{h}$ were significantly higher than the other groups (Table IV).

The patients who received heavy bupivacaine and morphine sulfate required more epidural analgesics in the postoperative period. However, this result did not constitute any statistically significant difference (Table V). The number of demands are seen in Table V. None of the patients required epidural analgesic solution more than 2 times. The duration of analgesia by isobaric bupivacaine with morphine was the longest time (Figure 3).

\section{Discussion}

Combined spinal epidural anaesthesia has been widely used by obstetric anaesthetists since the 1980s. More than $60 \%$ of consultant obstetric anaesthetists in England used the CSEA technique

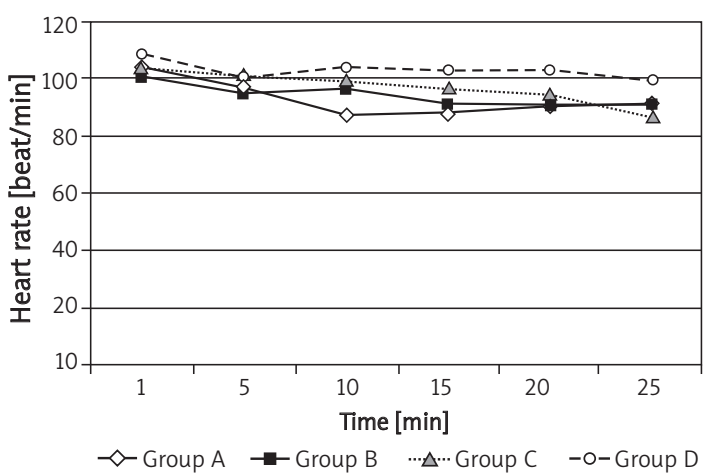

Figure 2 . Heart rate values after spinal blockade

Table II. Nausea and vomiting incidence (\%)

\begin{tabular}{|lcccc|}
\hline & Group A & Group B & Group C & Group D \\
\hline Yes & $3(12)$ & $2(8)$ & $2(8)$ & $2(8)$ \\
\hline No & $22(88)$ & $23(92)$ & $23(92)$ & $23(92)$ \\
\hline
\end{tabular}

$p>0.05$

during labour or caesarean section [13]. Combined spinal epidural anaesthesia allows for use of smaller doses of local spinal anaesthetics because the block can be supplemented at any time [14]. Compared to spinal block, the duration of the anaesthesia can be extended by using the CSEA technique. Combined spinal epidural anaesthesia also allows a reduction in the duration of the anaesthesia by the subarachnoid administration of the minimal dose of the local anaesthetic to establish the initial level for the surgery [15].

The duration of spinal anaesthesia depends on the characteristics of the local anaesthetics. Bupivacaine is a potent local anaesthetic with hydrophobic nature [16]. Both bupivacaine and morphine are characterised by a long duration of action. Dosage, volume and concentration of

Table III. Ephedrine requirement (mean \pm SD)

\begin{tabular}{|lcccc|}
\hline & Group A & Group B & Group C & Group D \\
\hline Ephedrine requirement $[\mathrm{mg}]$ & $10 \pm 9.57$ & $5.2 \pm 10.84$ & $6.8 \pm 9.45$ & $10.8 \pm 10.37$ \\
\hline$p>0.05$ & &
\end{tabular}

Table IV. Comparison of postoperative pain scores (VAS) (values are means)

\begin{tabular}{|lcccc|}
\hline Postoperative time [h] & Group A & Group B & Group C & Group D \\
\hline $1^{\text {st }}$ & $6.8 \pm 8.52$ & $8.4 \pm 8.50$ & $7.6 \pm 9.25$ & $5.6 \pm 8.20$ \\
\hline $2^{\text {nd }}$ & $24.8 \pm 15.30$ & $36 \pm 23.09$ & $27.2 \pm 14.86$ & $27.2 \pm 16.46$ \\
\hline $4^{\text {th }}$ & $25.2 \pm 20.84$ & $24.4 \pm 24.33$ & $20 \pm 21.99$ & $21.6 \pm 17.70$ \\
\hline $8^{\text {th }}$ & $4.4 \pm 7.68$ & $10.8 \pm 11.87^{*}$ & $5.2 \pm 6.53$ & $4.0 \pm 8.16$ \\
\hline $1^{\text {th }}$ & $9.2 \pm 11.15$ & $7.6 \pm 14.23$ & $11.2 \pm 14.23$ & $7.6 \pm 12.34$ \\
\hline $2^{\text {th }}$ & $7.2 \pm 6.78$ & $3.6 \pm 5.68$ & $7.6 \pm 7.23$ & $5.2 \pm 5.85$ \\
\hline
\end{tabular}

${ }^{*}$ Between groups $p<0.05$ 
Table V. Additional analgesic requirement

\begin{tabular}{|lcccc|}
\hline & Group A & Group B & Group C & Group D \\
\hline Yes & $1(4 \%)$ & $1(4 \%)$ & $3(12 \%)$ & $2(8 \%)$ \\
\hline No & $24(96 \%)$ & $24(96 \%)$ & $22(88 \%)$ & $23(92 \%)$ \\
\hline$p>0.05$ & & & &
\end{tabular}

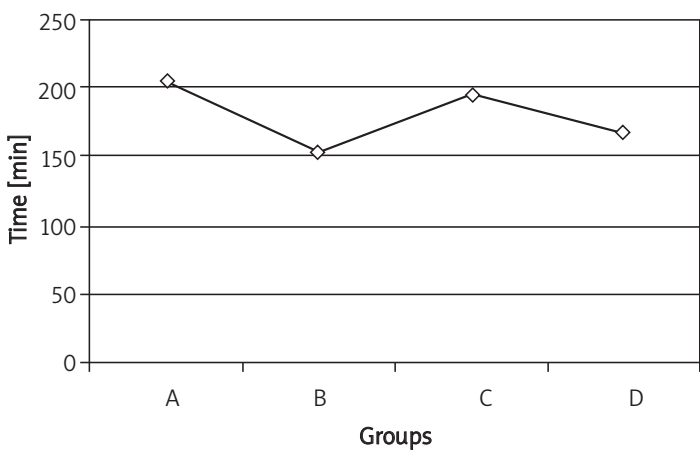

Figure 3. Postoperative first analgesic requirement time

anaesthetic are the major determinants of intrathecally administered solutions [17]. Also the analgesic effect of the additional opioids provides a long postoperative period without pain. As shown in several previous studies, the patients with morphine had a long duration of analgesia [9]. It was not a surprise for us that we had better results with morphine than fentanyl. However, there is always a potential risk of respiratory depression after neuraxial opioids. The incidence of respiratory depression after morphine administration ranges from $0 \%$ to $0.9 \%$. Late occurrence might be seen until the postoperative $18^{\text {th }} \mathrm{h}$ and can cause permanent brain damage [18]. Our postoperative repeated visits for sedation and respiratory rate monitoring were a precaution for early detection of respiratory depression and provide increased patient satisfaction. None of the patients had a respiratory rate less than 12 .

Ogun et al. [19] found that intrathecal isobaric ropivacaine $0.5 \% 15 \mathrm{mg}$ with morphine $150 \mu \mathrm{g}$ provided sufficient anaesthesia for caesarean delivery. The parturients were compared with the bupivacaine group. Time to first complaint of pain was similar in all groups.

In the study of Vercauteren et al. [20], they considered that the incidence of hypotension and ephedrine requirement with isobaric bupivacaine were more common than with heavy bupivacaine. The frequency of ephedrine requirement was similar in all groups in our study. However, the heart rate of patients in group A decreased significantly at the $10^{\text {th }} \mathrm{min}$ although the values remained within physiological limits. We thought that this result was coincidental and had no clinical significance because there was no statistically significant difference between groups in sensory levels and spread of anaesthesia and analgesia.

The duration of anaesthesia was shorter with hyperbaric bupivacaine compared with isobaric solution in a study by Malinovsly et al. [21]. We could not find any significant difference in the duration of spinal anaesthesia between groups.

The limitation of our study is that we administered only spinal block for the operation and we used the epidural route only for postoperative analgesia, so the amounts of local anaesthetics and opioids we used were higher than the conventional ones used for the CSEA technique. On the other hand, we primarily aimed to compare the effect of different baricities of bupivacaine with two different opioids and we demonstrated that the postoperative first analgesic requirement time was longer with isobaric bupivacaine than with heavy bupivacaine combined with morphine.

In conclusion, that intrathecal $15 \mathrm{mg}$ isobaric bupivacaine with $200 \mu \mathrm{g}$ morphine provides longer duration of analgesia, similar haemodynamic effects, ephedrine requirement and side effects when compared to heavy bupivacaine-morphine or bupivacaine-fentanyl combinations during caesarean section. Further studies can be planned with lower doses of morphine with different concentrations of bupivacaine when used with the CSEA technique.

\section{Acknowledgments}

The work was conducted in the Central Education and Research Hospital, Erzurum, Turkey.

\section{References}

1. Velde MV, Teunkens A, Hanssens M. Intrathecal sufentanil and fetal heart rate abnormalities: a double-blind, duble placebo-controlled trial comparing two forms of combined spinal epidural analgesia with epidural analgesia in labor. Anesth Analg 2004; 98: 1153-9.

2. Kamiya Y, Kikuchi T, Inagawa G. Lidocaine concentration in cerebrospinal fluid after epidural administration: a comparison between epidural and combined spinalepidural anesthesia. Anesthesiology 2009; 110: 1127-32.

3. Nakamura G, Ganem EM. Effects on mother and fetus of epidural and combined spinal-epidural techniques for labor analgesia. Rev Assoc Med Bras 2009; 55: 405-9.

4. Akbay KB, Boztepe A, Sezen YG. A comparison of bupivacaine solutions with or without fentanyl intrathecally. Türkiye Klinikleri J Med Sci 2007; 5: 118-23.

5. Malinovsky J, Renaud G, Le Corre, P. Influence of volume and baricity of solutions. Anesthesiology 1999; 9: 1260.

6. Kokki H, Tuovinen K, Hendolin H. Spinal anesthesia for paediatric day-case surgery: a double-blind, randomized, parallel group, prospective comparison of isobaric and hyperbaric bupivacaine. Br J Anaesth 1998; 81: 502-6.

7. Hallworth SP, Fernando R, Columb MO. The effect of posture and baricity on the spread of intrathecal 
bupivacaine for elective cesarean delivery. Anesth Analg 2005; 100: 1159-65.

8. Parlow JL, Money P, Chan P. Addition of opioids alters the density and spread of intrathecal local anesthetics? An in vitro study. Can J Anesth 1999; 46: 66-70.

9. Santos LM, Santos VCJ. Intrathecal morphine plus general anesthesia in cardiac surgery: effects on pulmonary function, postoperative analgesia and plasma morphine concentration. Clinics 2009; 64: 279-85.

10. Andrieu G, Roth B, Ousmane L. The efficacy of intrathecal morphine with or without clonidine for postoperative analgesia after radical prostatectomy. Anesth Analg 2009; 108: 1954-7.

11. Channaiah VB, Chary K, Vlk JL, Wang Y, Chandra SBC. Lowdose fentanyl: hemodynamic response to endotracheal intubation in normotensive patients. Arch Med Sci 2008; 4: 293-9.

12. Eisenach J, Hood D, Curry R. Cephalad movement of morphine and fentanyl in humans after intrathecal injection. Anesthesiology 2003; 99: 166-173.

13. Ingelmo PM, Somaini M. Optimal epidural volume expansion during combined spinal-epidural anesthesia: one question, one answer. Minerva Anesthesiol 2010; 76: 334-9.

14. Nakamura G, Ganem EM. Effects on mother and fetus of epidural and combined spinal-epidural techniques for labor analgesia. Rev Assoc Med Bras 2009; 55: 405-9.

15. Imbelloni LE, Fornasari M. Combined spinal epidural anesthesia during colon surgery in a high-risk patient. Case report. Rev Bras Anestesiol 2009; 59:741-5.

16. Raddi P, Nagalingaswamy VP, Khatib F, Wang Y, Chandra SBC. A comparison of interpleural bupivacaine and intravenous pethidine for postoperative pain relief following open cholecystectomy. Arch Med Sci 2009; 5: 57-62.

17. Kuusniemi KS, Pihlajamäki KK, Pitkänen MT. Low dose bupivacaine: a comparison of hypobaric and near isobaric solutions for arthroscopic surgery of the knee. Anaesthesia 1999; 54: 540-5.

18. Carvalho B. Respiratory depression after neuraxial opioids in the obstetric setting. Anesth Analg 2008; 107: 956-61.

19. Ogun CO, Kirgiz EN, Duman A. Comparison of intrathecal isobaric bupivacaine-morphine and ropivacaine-morphine for caesarean delivery. Br J Anaesth 2003; 90: 659-64.

20. Vercauteren MP, Coppejans HC, Hoffmann VL. Small-dose hyperbaric versus plain bupivacaine during spinal anesthesia for cesarean section. Anesth Analg 1998; 86: 989-93.

21. Malinovsky JM, Géraldine R, Pascal LC. Intrathecal bupivacaine in humans: influence of volume and baricity of solutions. Anesthesiology 1999; 91: 1260. 